\title{
Comparation of LoRa and NB-IoT
}

\author{
Yanhui $\mathrm{Ji}^{1} \&$ Hao Yang ${ }^{2}$ \\ ${ }^{1}$ School of Information Engineering, Yancheng Teachers University, Yancheng, China \\ ${ }^{2}$ Jiangsu Provincial Key Constructive Laboratory for Big Data of Psychology and Cognitive Science, Yancheng \\ Teachers University, Yancheng, China \\ Correspondence: Hao Yang, Jiangsu Provincial Key Constructive Laboratory for Big Data of Psychology and \\ Cognitive Science, Yancheng Teachers University, Yancheng, China
}

Received: December 1, 2018 Accepted: January 24, 2019 Online Published: January 30, 2019

doi:10.5539/nct.v4n1p13 URL: https://doi.org/10.5539/nct.v4n1p13

\begin{abstract}
When people use Internet of Things (IoT), they should consider many factors, such as node cost, network cost, battery life, data transmission rate, delay, mobility, network coverage and deployment type. It can be said that no technology can satisfy all the needs of IoT. NB-IoT and LoRa technologies have different technical and commercial characteristics, so they differ in application scenarios. In this paper, the differences between the two will be elaborated.
\end{abstract}

Keywords: networks, LoRa, NB-IoT

\section{Introduction of LoRa}

LoRa (Long Distance) is a novel technology and developed by Semtech. Its typical operating frequency is 915 MHz in the United States, $868 \mathrm{MHz}$ in Europe and $433 \mathrm{MHz}$ in Asia. LoRa's Physical Layer (PHY) uses a unique form of FM chirped spread spectrum technology with forward error correction (FEC). This spread spectrum modulation allows multiple radio devices to use the same frequency band as long as each device uses different chirps and data rates. Its typical range is $2 \mathrm{~km}$ to $5 \mathrm{~km}$, and the longest distance can reach $15 \mathrm{~km}$, depending on the location and antenna characteristics.

\section{Introduction of NB-IoT}

Narrow-Band Internet of Things (NB-IoT) has become an important branch of the Internet of Things. NB-IoT is built in cellular network and needs a narrow bandwidth about $180 \mathrm{KHz}$. It can be directly deployed in GSM network, UMTS network or LTE network to reduce deployment cost and achieve smooth upgrade. NB-IoT is a new technology in the field of IoT. It supports low power devices'cellular data connection in WAN. It is also called low power wide area network (LPWAN). NB-IoT supports efficient connection of devices with long standby time and high network connection requirements. It is said that the battery life of NB-IoT devices can be increased by at least 10 years, while providing a very comprehensive coverage of indoor cellular data connections.

\section{Frequency Band, Quality of Service and Cost}

LoRa works in an unauthorized frequency band below $1 \mathrm{GHz}$, so there is no additional charge for its application. NB-IoT and cellular communications use authorized bands below $1 \mathrm{GHz}$. The frequency band between $500 \mathrm{MHz}$ and $1 \mathrm{GHz}$ is the best choice for long-distance communication, because the actual size and efficiency of the antenna have considerable advantages.

LoRaWAN uses free unauthorized frequency bands and is an asynchronous communication protocol, which is the best choice for battery power supply and low cost. LoRa and LoRaWAN protocols have unique characteristics in dealing with interference, network overlap and scalability, but they can not provide the same quality of service (QoS) as cellular protocols. Authorized bidding for the Sub-GHz band is known to cost more than $\$ 500$ million per MHz. Cellular networks and NB-IoT do not provide the same battery life as LoRa due to quality of service (QoS) considerations. Due to the high bandwidth usage cost and QoS, it is necessary to ensure that cellular networks and NB-IoT are recommended for the application scenarios of QoS, and LoRa is a good choice if low cost and lots of connections are preferred. 


\section{Battery Life and Downlink Delay}

The concept of cellular network design is optimal bandwidth utilization, which sacrifices node cost and battery life accordingly. On the contrary, LoRaWAN nodes are designed for low cost and long battery life, and have some shortcomings in band utilization.

There are two important factors to consider in terms of battery life, current consumption (peak current and average current) of nodes and protocol content. LoRaWAN is an asynchronous ALOHA-based protocol, that is to say, nodes can sleep long or short according to specific application scenarios, while nodes with synchronization protocols such as cellular networks must be connected regularly. For example, mobile phones on the market now have to synchronize with the network every $1.5 \mathrm{~s}$. In NB-IoT, this synchronization decreases but still occurs on a regular basis, which consumes extra battery power.

Modulation is an effective way to make full use of frequency band in cellular networks, but it is not effective from the point of view of nodes. Cellular modulation (OFDM or FDMA) requires a linear transmitter to generate modulation signals, and a linear transmitter needs several orders of magnitude more peak current than a non-linear modulation. The higher the peak current, the more power the battery will consume.

However, synchronous communication protocols have advantages in shorter downlink latency, and NB-IoT can provide fast data transmission rate for applications requiring a large amount of data throughput. Class B of LoRaWAN shortens the downlink communication delay by periodically (programmed) waking up the terminal to receive downlink messages.

So NB-IoT may be a better choice for applications requiring frequent communications, shorter latency or larger data volumes, while LoRa is better for scenarios requiring lower costs, higher battery life and less frequent communications.

\section{Network Coverage and Deployment Schedule}

The essential requirement of node work is network coverage. For NB-IoT, an obvious advantage is that network deployment can be provided by upgrading existing network facilities, but this upgrade is limited to some specific 4G/LTE base stations, and the cost is high. And this upgrade is only suitable for urban areas that already have $4 \mathrm{G} / \mathrm{LTE}$ coverage. It is not suitable for remote or suburban areas where there is no $4 \mathrm{G}$ coverage. The NB-IoT standard was released in June 16, and the module is expected to be mass-produced in the first half of 17 years. In addition to network deployment, the corresponding commercialization and the establishment of industrial chain need more time and effort to explore, but will market demand and opportunities wait?

LoRa's entire industrial chain is relatively mature, and its products are in a "ready" state. At the same time, many countries around the world are carrying out or have completed national network deployment. A prominent advantage of the LoRa industry chain is that members of each sector have autonomy, and some large companies are planning to create a hybrid business model to deploy networks and applications. However, NB-IoT industry chain will be limited by frequency band, operators and so on.

\section{Equipment Cost, Network Cost and Hybrid Model}

For terminal nodes, LoRaWAN protocol is simpler than NB-IoT, easier to develop, and better applicable and compatible for microprocessors. The modulation mechanism and protocol of NB-IoT are more complex, which requires more complex circuits and more cost. At the same time, NB-IoT is taxable as 3GPP. At this stage, the tax for a mobile phone is about $\$ 5$, but it seems too expensive for Internet of Things devices, and the price chaos in mobile communication market such as mobile phones will be caused if the tax is reduced rashly. So how to balance the tax and fee between IoT and mobile communication is also a big problem for $3 \mathrm{GPP}$ organizations.

LoRa modules with low cost and relatively mature technology can be found in the market, and upgrades will follow. The LoRa Alliance does not have too many copyright and tax restrictions, making it considerable to have modules under $\$ 4$ in the LoRa industry chain. Nowadays, the price of the LoRa module in the market is usually between $\$ 7$ and $\$ 10$, but it's not a big problem to raise $\$ 4$ to $\$ 5$ as technology matures. Now an LTE module can hardly be cheaper than $\$ 20$.

For the development of both IoT and LPWAN, different models are required to reduce expenditure and operating costs, compared with the traditional "tower" network. LoRaWAN deployment costs less because traditional towers, industrial base stations and even portable home gateways can be used. At this stage, the price of a tower base station is about $\$ 1,000$, the price of an industrial base station is less than $\$ 500$, and the price of a home gateway is only about $\$ 100$. But for NB-IoT, the price of upgrading existing 4,000 LTE base stations is conservatively estimated at no less than $\$ 15,000$. 


\section{Acknowledge}

This work is supported by National Natural Science Foundation of China under Grant No. 61772448, 61402394, Natural Science Major Project of the Higher Education Institutions of Jiangsu Province of China under Grant No. 17KJA520006, Jiangsu Provincial Key Constructive Laboratory for Big Data of Psychology and Cognitive Science under Grant No. 72591862004G, Six talent peaks project in Jiangsu Province under Grant No. JY-072, 333 high level talent training project In Jiangsu Province, and sponsored by Qing Lan Project.

\section{References}

Bankov, D., Khorov, E., \& Lyakhov, A. (2017, June). Mathematical model of LoRaWAN channel access. In Proceedings of the IEEE 18th International Symposium on A World of Wireless, Mobile and Multimedia Networks (WoWMoM), Macao, China (pp. 12-15). http://doi.org/10.1109/WoWMoM.2017.7974300

Hejazi, H., Rajab, H., Cinkler, T., \& Lengyel, L. (2018, January). Survey of platforms for massive iot. In Future IoT Technologies (Future IoT), 2018 IEEE International Conference on (pp. 1-8). IEEE.

Lin, J., Shen, Z., \& Miao, C. (2017, July). Using blockchain technology to build trust in sharing LoRaWAN IoT. In Proceedings of the 2nd International Conference on Crowd Science and Engineering (pp. 38-43). ACM. https://doi.org/10.1145/3126973.3126980

Mekki, K., Bajic, E., Chaxel, F., \& Meyer, F. (2018). A comparative study of LPWAN technologies for large-scale IoT deployment. ICT Express. Retrieved from https://hal.archives-ouvertes.fr/hal-01670379

Sinha, R. S., Wei, Y., \& Hwang, S. H. (2017). A survey on LPWA technology: LoRa and NB-IoT. Ict Express, 3(1), 14-21. https://doi.org/10.1016/j.icte.2017.03.004

\section{Copyrights}

Copyright for this article is retained by the author(s), with first publication rights granted to the journal.

This is an open-access article distributed under the terms and conditions of the Creative Commons Attribution license (http://creativecommons.org/licenses/by/4.0/). 